\title{
Protocolo previo al inicio de técnicas de reproducción asistida
}

\author{
José Ignacio Madero C. M.D.*; Patricio Neira M.D.; Pilar Gaitán M.D. MsC.; Eduardo Sanz BSc; Luz Mabel Avila \\ MsC; Magaly Escobar MsC; Jesús Alberto Ruiz M.D
}

\section{RESUMEN}

La adecuada selección y estudio de las parejas que ingresan a los Programas de Reproducción Asistida garantiza los resultados en cuanto a tasas de embarazo y bebes en casa. La preparación de estas parejas consta en la mujer de un estudio cuidadoso de la función endocrina como es el conocer la reserva ovárica, el descartar patología utero-tubárica como el hidrosalpinx, la endometriosis, alteraciones del canal endocervical o de la cavidad endometrial. En el varón la presencia de alteraciones de los parámetros seminales pueden conducir desde una falla en la fertilización hasta infección en los sistemas de cultivos, y su cuidadosa evaluación optimiza los resultados. Siguiendo este protocolo estricto hemos logrado incrementar de manera importante nuestras tasas de gestación en un $13 \%$ (de $21 \%$ en 1995 y 1996 a $34 \%$ en 1997 ) por ciclo, lo que nos motiva a presentarlo.

PALABRAS CLAVES: Fertilización in vitro, téenicas de reproducción asistida, transferencia de embriones, endometriosis, infertilidad masculina, análisis de semen, pruebas funcionales espermáticas.

\section{SUMMARY}

The strict criteria for the selection and study of the couples that ingress to the Reproductive Assisted Programs guarantees the results in pregnancy rates and baby at home. The preparation of these couples, in women consist on a carefully study of the endocrine function like knowing the ovarian reserve, letting out uterine and tubule pathologies like the hydrosalpinx, endometriosis, alterations of the cervical channel or endouterine cavity. In the male the presence of any alteration of the seminal parametres, can conduce to fertilization failure infection of the culture system, and a careful evaluation optimized the results. Following these strict protocol in these couples, we improve is an important manner our pregnancy rate in $13 \%$ (21\% in 1995 and 1996 to $34 \%$ in 1997$)$ per cycle.

KEY WORDS: Assisted reproductive techniques, infertility work-up, endometriosis, embryo transfer techniques, male infertility, semen analysis, sperm functional test, in vitro fertilization.

\section{Introducción}

La evaluación adecuada de las parejas con problemas de fertilidad sigue siendo la clave para el éxito del tratamiento. Se inicia con una detallada Historia Clínica donde la edad de la pareja y la duración de la infertilidad son dos puntos fundamentales para la toma de decisiones en cuanto a estudios diagnósticos, que incluyen una serie de determinaciones hormonales, test dinámicos y estudios específicos que conducirán al inicio de una terapéutica definitiva que, en lo posible, ofrezca a la pareja infértil unas posibilidades de éxito cercanos a la media mundial (1).

\section{Materiales y métodos}

Desde el año 1996 nuestro equipo diseñó un protocolo de estudio y manejo de las parejas que ingresan a cualquier procedimiento de reproducción asistida con base en

\footnotetext{
* Director Científico. MEDiferTIL. Programa de Medicina Reproductiva.
}

el análisis de la literatura mundial utilizando la metodología de la evidencia comprobada.

El objetivo de esta publicación es mostrar el protocolo que se implementó en nuestro centro con el propósito de incrementar las tasas de gestación. Analizamos los resultados de 677 ciclos en los cuales se utilizaron técnicas de reproducción asistida durante el período comprendido entre enero de 1995 y diciembre de 1997 y que fueron clasificados en dos grupos.

1.387 pacientes sometidas a fertilización in vitro durante el período comprendido entre enero de 1995 a diciembre de 1996 a las cuales no se les aplicó el protocolo. 2.290 pacientes a quienes se les aplicó el protocolo durante el año de 1997. Nuestro protocolo consiste en una adecuada valoración y preparación de la pareja siendo estudiados así:

\section{Evaluación de la mujer}

Entre los factores más importantes que tienen una relación directa con los resultados de los tratamientos de fertilidad está la edad de la mujer (2) y su relación con el envejecimiento ovárico, el cual en la mayoría de los 
casos se presenta por una disminución del pool de folículos ováricos, disminución de los niveles de inhibina y elevación de los niveles de la hormona estimulante de los folículos (FSH), con la consecuente elevación de los niveles basales de estradiol $(3,4)$. Esto produce la aparición de picos prematuros de hormona luteinizante ( $\mathrm{LH}$ ) y ovulación, o en otros casos a luteinización temprana. Es aquí cuando se observa una tasa de crecimiento folicular acelerado que lleva a un acortamiento de la fase folicular y del ciclo menstrual. Los ovarios de estas mujeres con una reserva disminuida tienen pocos folículos y estos están en un medio hipóxico, con pocas células de la granulosa, y una función disminuida en cuanto a producción de esteroides y glucoproteínas, presentan disminución de la mitosis y aumento de la apoptosis (5-7). De otro lado se encuentra una disminución de la calidad ovocitaria con aumento de las anormalidades genéticas en sus embriones, independientemente de los niveles de FSH lo que se refleja en un mayor número de fallos en la implantación y un aumento en la tasa de abortos (8-10).

Tan importante en la edad como la evaluación de la reserva funcional ovárica, que se hace determinando los niveles basales de FSH en la fase folicular temprana, días 2-5 del ciclo, lo que permite determinar las dosis de inicio de los medicamentos y tratar de predecir la respuesta al mismo. Está demostrado que niveles de FSH mayores de $15 \mathrm{mUL} / \mathrm{ml}$ están asociados con una mala respuesta a la estimulación ovárica $(11,12)$ y una tasa muy baja de gestación (13). En algunas mujeres mayores, la maduración folicular es más rápida, presentando una disminución de los niveles de FSH en el día 3-4; en estos casos se debe correlacionar con los niveles basales de estradiol, los cuales no deben ser mayores de $50 \mathrm{pgr} / \mathrm{ml}$, y nos ayudan a identificar aquellas pacientes que a pesar de tener niveles normales de FSH responden mal a la estimulación ovárica (13). Además de la pobre respuesta a la inducción de la ovulación, está demostrado que los niveles elevados de FSH están asociados, aún en mujeres menores de 40 años a una tasa mayor de anormalidades cromosómicas (8), lo que hace a al FSH un marcador en el proceso ovárico de pérdida de la reserva ovocitaria asociado a una tasa de embarazos muy baja.

Cuando existe la sospecha de una falla ovárica oculta y la paciente refiere el antecedente de lesiones como endometriomas o cirugía ovárica es conveniente realizar el test de clomifeno $(14,15)$ el cual consiste en la administración de $100 \mathrm{mg}$ diarios de citrato de clomifeno del quinto al noveno día del ciclo menstrual, midiendo previamente los niveles basales de FSH y en el día 11 del ciclo. Cuando se suman estos valores y se encuentran elevados (mayores de $26 \mathrm{mUI} / \mathrm{ml}$ ) se confirma la falla ovárica; se puede correlacionar también con los niveles de estradiol basal y en día 11 los que al sumarlos son más bajos que los de las pacientes normales. Una posible explicación de la respuesta de la FSH al clomifeno podría ser el bajo nivel de inhibina secretado por los folículos ováricos que ya tienen una alteración funcional.

Cuando la determinación de los niveles basales de LH (día 3 ) es menor de $3 \mathrm{mUl} / \mathrm{ml}$, está asociada a una mala respuesta a la inducción de ovulación, y si el cociente FSH/LH es mayor de 3, se relaciona con un aumento de cancelaciones de ciclos de Fecundación In Vitro (FIV) (16).

Otro test dinámico como el del Acetato de Leuprolide (17) o el EFORT (18) no aportan más que la determinación de los niveles basales de FSH y Estradiol.

De otro lado debemos considerar que la presencia de alteraciones uterinas, tubáricas, así como las producidas por la endometriosis, suman un $45 \%$ de las parejas que ingresan a los programas de reproducción asistida (1), por lo que debemos comprobar su normalidad en primera instancia, con la ecografía transvaginal, la cual identifica alteraciones uterinas como malformaciones, pólipos, leiomiomas, adenomiosis, o patología tubárica como el hidrosalpinx, además valoramos el tamaño de los ovarios, la presencia de folículos antrales en fase folicular temprana y su medición, lo que nos ayuda a predecir la respuesta a la inducción de ovulación (19). Con la aparición del Doppler color es posible estudiar el ovario en sus fases de reclutamiento, selección, dominancia y ovulación, permitiéndonos conocer su estado fisiológico, así como las patologías que se presenten (20).

La Histerosalpingografía es de gran utilidad para evaluar la presencia de lesiones dentro de la cavidad uterina, que impiden la implantación luego de la transferencia embrionaria, así como el estado de las trompas de Falopio. Es importante descartar la presencia de hidrosalpinx y diferenciarlo de imágenes normales por sobredistensión.

La Histeroscopia diagnóstica y/o operatoria debe realizarse casi de rutina antes de iniciar los procedimientos de reproducción asistida, ya que nos permite detectar y corregir alteraciones a nivel del cérvix y endocérvix, principalmente estenosis, sinequias e infecciones (21) o de cualquier patología intrauterina como pólipos, miomas submucosos y sinequias. Se puede realizar concomitantemente con la Laparoscopia, y permite conocer exactamente el estado de útero y anexos, la relación fimbria con el ovario y la presencia de patologías importantes como la endometriosis, presente en el $15 \%$ de las mujeres que ingresan a los programas de reproducción asistida (1). Una vez diagnosticada la endometriosis, la clasificamos de acuerdo con los criterios de la última revisión de la Sociedad Americana de Medicina Reproductiva (22) en: Estado I (mínima) y II (leve) están caracterizadas por la presencia de implantes superficiales peritoneales u ováricos, con o sin adherencias laxas; los Estados III (moderada) y IV (severa) usualmente tienen implantes ováricos profundos, adherencias densas o fondo de saco posterior sellado (22). La endometriosis debe ser tratada durante el mismo procedimiento mediante la resección/ablación de los focos, liberación de adherencias y sí existen lesiones ováricas tipo endometriomas resecarlos. Además de las alteraciones anatómicas que produce la endometriosis en sus estadios III y IV, como la pérdida de la relación fimbria-ovario, en los estadios I y II, se ha planteado que la infertilidad es producida por elevación en la concentración de prostaglandinas a nivel peritoneal, que interfieren con la ovulación (23-24), alteran el peristaltismo tubárico, la función del cuerpo lúteo, disminuyen la movilidad espermática o aumentan la contractilidad uterina interfiriendo con la implantación del blastocisto. La endometriosis se asocia también con la 
presencia de una mayor concentración de macrófagos peritoneales, lo que aumenta el riesgo de fagocitosis de espermas (25); por último una alteración de la autoinmunidad (26) y una deficiente inmunidad celular puede jugar un papel importante en la infertilidad asociada a la endometriosis (27).

Otras pruebas que deben tenerse en cuenta en la evaluación femenina antes de iniciar una fecundación in vitro son:

Capacidad funcional del cérvix uterino: la ecografía transvaginal, la histerosonografía y la histerosalpingografía son de gran ayuda para valorar el tamaño y características del canal endocervical. Aun tiene validez la prueba postcoital, pues ayuda a discriminar de una manera general problemas a este nivel; se debe realizar en etapa preovulatoria y para ello es mejor guiarse por la ecografía seriada transvaginal; un resultado negativo nos orienta hacia una infección genital en uno o ambos miembros de la pareja, a la presencia de anticuerpos antiespermatozoides, problemas en el semen que deben reflejarse en el espermograma, o deficiente secreción de moco cervical, como resultado de lesiones locales, la existencia de hipoestrogenismo o infecciones. Está demostrado que los microorganismos a nivel cervical pueden afectar negativamente el resultado de la fecundación in vitro por propagación de la infección al momento de la transferencia embrionaria (21).

Anticuerpos Anti-chlamydia: siempre que se encuentre un daño tubárico debe descartarse la presencia de secuelas de infección por $C$. trachomatis, además su presencia altera la implantación y aumenta la tasa de abortos debido a una posible infección endometrial crónica o a efectos permanentes por la misma, por mecanismos autoinmunes; también produce ruptura prematura de membranas, bajo peso al nacimiento y muerte intrauterina. Un cultivo cervical negativo no descarta este tipo de infección, debiendo recurrirse a técnicas de diagnóstico más precisas como la reacción en cadena de la polimerasa (PCR). El tratamiento a la pareja debe realizarse con Azitromicina un gramo oral, dosis única que reemplaza el tradicional de Doxiciclina por siete días, y como régimen alternativo está la Eritromicina Base 500 mgrs. cuatro veces al día durante cinco días o la Ofloxacina 200 mgrs. dos veces al día por siete días (28).

Ensayo de transferencia (Dummy o Mock): de vital importancia en todas las pacientes que van a ingresar a cualquier procedimiento de reproducción asistida, pues nos permite conocer previamente el canal endocervical, saber si hay estrecheces o dificultades para tenerlas en cuenta el día de la transferencia; se esquematiza en la hoja de seguimiento folicular y en la historia clínica las características del cérvix, el tamaño uterino, la profundidad del cérvix, para decidir con anterioridad el tipo y marca de catéter a utilizar para la transferencia embrionaria: con camisa, con guía metálica, blando, etc. (29-31). Se ha demostrado que la adecuada selección de los catéteres mejora las tasas de embarazos. (32-33).

\section{Evaluación del varón}

La frecuencia de las alteraciones masculinas llega al $23 \%$ dentro de las parejas que llegan a reproducción asistida (1) y su evaluación y estudio es tan importante como el de la mujer, siendo quizás el más fácil de iniciar mediante el espermograma básico, el cual debe solicitarse en la primera consulta; en caso de ser anormal se repetirá para confirmar el diagnóstico y será el punto de partida para una serie de estudios como son: Morfología espermática de acuerdo con los criterios estrictos de Kruger (34): es importante utilizarla pues está demostrado que la tasa de fertilización se encuentra disminuida en las muestras con morfología anormal: en el grupo de 0 a $4 \%$ de formas normales la tasa de fertilización es del 7,6\% comparada con el $63 \%$ en el grupo con morfología normal entre el 5 y el 14\% (34). Lo anterior se confirmó en otro estudio (35) donde se halló que la tasa de fertilización con morfología normal mayor del $14 \%$ era mayor del $80 \%$. Esto mismo ha sido demostrado por numerosos autores y confirmado en una extensa revisión por Coetzee (36) donde se concluye que la morfología espermática es el parámetro de mayor importancia en el análisis básico de semen y punto de partida en la elección del tipo de procedimiento a realizar.

Tinción para células blancas: la presencia de leucocitospermia y piospermia son indicativas de procesos inflamatorios a nivel de las glándulas accesorias principalmente y se acompañan de bacteriospermia. Siempre se debe realizar un espermocultivo para confirmar el tipo de germen y poder iniciar una terapéutica dirigida a la infección. Con esto garantizamos que no se produzcan ni contaminaciones de los cultivos embrionarios ni el efecto deletéreo de metabolitos tóxicos bacterianos sobre los espermas y no aparezcan los radicales oxígeno reactivos (ROS) generados por la presencia de células blancas, principalmente neutrófilos. Los ROS son producidos en niveles bajos o fisiológicos; moléculas como el Peróxido de Hidrógeno son muy importantes en el proceso de capacitación de los espermatozoides, pero cuando se producen en grandes cantidades (37), los ROS alteran la función espermática por daño peroxidativo en la membrana plasmática del espermatozoide, generando una ruptura de las cadenas de DNA. Este daño se produce por existir una limitada producción de las enzimas antioxidantes citoplasmáticas (superóxido dismutasa, catalasa, glutatión peroxidasa) en el espermatozoide y de otro lado, un aumento de los substratos para el daño oxidativo (ácidos grasos no saturados y DNA). Como consecuencia, la generación de ROS por el lavado del eyaculado ha demostrado tener una correlación negativa con las tasas de fertilización in vivo e in vitro (38). De otro lado la presencia de bacterias en el eyaculado se asocia a una disminución en su capacidad fertilizante y está relacionado directamente con una disminución en el número y movilidad de los espermatozoides, asi como un aumento importante de las formas anormales (39-40) ya sea por efecto de sustancias metabólicas tóxicas o algún tipo de eferto directo de las bacterias sobre los espermas, pues se han podido observar pegadas a la superficie de los espermatozoides (41). Existe al parecer, una correlación directa en el eyaculado entre la presencia de células blancas y bacterias, pero no de células redondas y bacterias (40).

Prueba de penetración espermática (SPA): valora la disposición del espermatozoide humano respecto a los procesos de capacitación, reacción acrosómica y fusión 
con la membrana vitelina del ovocito. Sus resultados se correlacionan muy bien con los resultados del FIV y el embarazo espontáneo pero tiene inconvenientes: su dificultad técnica, el tiempo que requiere, y la estandarización. De todos modos es una prueba de gran sensibilidad para la medición de la función espermática y la capacidad luego de realizada la reacción acrosómica de iniciar la función esperma-ovocito (37).

Prueba de la hemizona (HZA): en los pacientes que presentan teratozoospermia la unión a la zona pelúcida y posterior fertilización se encuentra alterada (42); cuando se comparó con la población normal en FIV, en los teratozoospérmicos se observó una unión a un nivel diferente, así como una disminución en los resultados en la prueba ( $36 \pm 7$ vs $10 \pm 3$ ). Lo anterior ha sido confirmado (43) al aumentar en los pacientes con teratozoospermia severa el número de espermatozoides inseminados por ovocito, de 50.000 a 500.000 por ml, dando como resultado un incremento en la tasa de fertilización (44). Existe una excelente correlación entre la prueba de la hemizona y los resultados del FIV concluyéndose que los resultados expresados como el índice de hemizona y la relación de los espermas unidos a la zona, en relación con la tasa de fertilización son significativamente correlacionables (45).

Reacción Acrosómica (RA): proceso de exocitosis que involucra la fusión de la membrana plasmática y la membrana acrosomal externa en la que juega un papel importante el flujo de calcio. Sólo los espermas que han reaccionado pueden penetrar la zona pelúcida. Los pacientes que presentan una alta incidencia de formas anormales. tienen una baja incidencia de RA espontánea y una respuesta disminuida a la estimulación con Progesterona, pero conservan una respuesta no específica al ionóforo calcio $(\mathrm{Ca}++)$ i. Se han observado anormalidades paralelas (ca++)i en estos pacientes con teratozoospermia, sugiriendo que en estas poblaciones de espermas puede existir un defecto no genómico del receptor de la Progesterona y/o anormalidades de otros sistemas de transducción de membrana (46). Una manera sencilla de valorar la reacción acrosómica se hace mediante tinciones supravitales.

Prueba de penetración espermática en ovocito de Hamster: examina la aptitud del espermatozoide humano para la capacitación, reacción acrosómica y fusión con el ovocito. Desde el punto de vista clínico se correlaciona muy bien con el resultado del FIV y el embarazo espontáneo. Tiene como desventaja el tiempo que requiere, así como las dificultades técnicas y de estandarización (47). Actualmente no lo estamos realizando pero reconocemos su relación con la capacidad de entrar en RA y los parámetros de movilidad que juntos nos evalúan el potencial de fertilidad de una muestra.

Creatinin Kinasa (CK): es un marcador celular de la calidad espermática, y clave en la síntesis de factores transportadores de energía. Niveles elevados indican un defecto en la extrusión citoplasmática. Se ha observado que las muestras de oligozoospérmicos luego de ser sometidas a swim-up, presentan un nivel más bajo de CK que la muestra original. En los FIV se puede predecir con base en la actividad de la CK, los grupos que presentaran buena fertilización (48). Por otro lado se halló que solamente los espermas que presentan un patrón maduro de inmunohistoquímica para $\mathrm{CK}$, selectivamente se unen a la zona pelúcida, a diferencia de los espermas con un patrón inmaduro para CK que tienen una deficiente capacidad para reconocer y unirse al ovocito (48).

Anticuerpos Antiespermatozoides (ASA): cuando están presentes en la mujer no se puede utilizar su suero para los medios de cultivo embrionario, ya que alteran la fertilización. Esto se evita utilizando suero sintético humano (no se debe utilizar suero bovino). En los casos de fallos previos en la fertilización, se debe descartar la presencia de los anticuerpos en el líquido folicular y realizar lavados extras de los cúmulos así como aumentar el número de espermas a inseminar. Para comprobar su presencia utilizamos semen del esposo contra suero de la esposa y un control negativo, con los inmunobead (49). Los ASA en el varón pueden resultar de una infección, trauma, cirugía o también aparecer sin ningún antecedente. Cuando la unión de $\operatorname{IgG}$ e $\operatorname{Ig} \mathrm{A}$ es mayor del $70 \%$ existe un alto chance de fallo en la fertilización, lo que se corrige aumentando el número de espermas inseminados, o diluyendo la muestra en medio de cultivo con suero al 50\% libre de anticuerpos. Finalmente, la Inyección Intracitoplasmática de Espermatozoide (ICSI) ha demostrado ser la mejor alternativa cuando se presentan estos casos (50).

Por la complejidad de algunas de las pruebas antes mencionadas, a pesar de reconocer su importancia y validez en el estudio del varón, únicamente estamos realizando el espermograma con criterios estrictos de Kruger, tinciones para células blancas y supravital para reacción acrosómica, y la detección de anticuerpos antiespermatozoides.

Otros aspectos a tener en cuenta son:

Disfunción sexual: en repetidas ocasiones nos hemos encontrado ante la dificultad del esposo para tomarse la muestra de semen, en especial el día de la aspiración folicular. En la consulta inicial se debe indagar acerca de este tipo de alteración, y comentar con la pareja cuando lo amerite, la necesidad de congelar por lo menos una muestra antes de la aspiración.

Enfermedades de transmisión sexual: en todas las parejas debemos realizar exámenes de laboratorio para descartar HIV, Hepatitis B, C y Sífilis. Lo anterior como medidas de seguridad para el personal que maneja los laboratorios, para la pareja y del futuro hijo.

Es también un requisito de las autoridades de salud locales y nacionales.

Hábitos personales: se debe interrogar acerca de la ingestión de tóxicos como marihuana, alcohol, cocaína, o de cualquier tipo de medicamento que pudiera causar alteraciones en la espermatogénesis. Recientemente se publicó un meta-análisis que valoró el impacto del cigarrillo en los resultados de la fertilización in vitro hallándose un efecto negativo (odds ratio 0.54) al aumentar la tasa de abortos (51) por lo que se aconseja a todas las parejas el suspenderlo con antelación.

\section{Resultados}

El análisis de nuestros resultados en 1998, mostró un aumento en las tasas de embarazo de un $21 \%$ en los años 1995 y 1996 a un $34 \%$ por ciclo en 1997 , y la tasa de 
nacimiento del $17,5 \%$ al $28 \%$, lo que nos motiva a presentarlo.

\section{Conclusiones}

Los resultados del presente reporte observacional descriptivo demuestran la importancia de seguir un protocolo estricto en las parejas que son sometidas a procedimientos de reproducción asistida, brindándoseles una selección de tratamiento más adecuada, y una mayor seguridad y efectividad en cuanto a los resultados. Así mismo es importante que la pareja conozca las interpretaciones de cada una de las pruebas que se estén realizan- do ya que esto los ayuda a conocer la severidad y complejidad del problema, comprender la solución propuesta, conocer los resultados y tener presente el índice de éxitos y fracasos. En conjunto se evaluarán los riesgos y beneficios del mismo y su elección será más fácil de realizar. Conociendo previamente las posibilidades reales de los diferentes tratamientos, se obtiene una mayor colaboración de las parejas y se facilita la aprobación del tratamiento propuesto. Al seguir el protocolo de estudios previos a cualquiera de las técnicas de reproducción asistida estamos garantizando un trabajo de calidad y optimizando los resultados.

\section{BIBLIOGRAFIA}

1. Center Diseases Control Reports: Results in ART 1996 National Summary. CDC. 1998. En: http: //webdev.nced.cdc.gov/dev/art96/ clinics96.asp.

2. Menken J.. Trussel J.. Larsen U. Age and infertility. Science 1986; 233: $1389-1394$.

3. Tatcher SS.. Naftolin F. The agin and aged ovary. Sem Reprod Endocrinol 1991; 9: 189-196.

4. Klein NA.. Battaglia DE., Fujimoto VY. et al. Reproductive aging: accelerated ovarian follicular development associated with a monotropic FSH rise in a normal older women. J Clin Endocrinol Metab 1996; 81: 1038-1045.

5. Friedman CI., Danforth DR., Herbosa EC et al. Folicular fluid vascular endothelial growth concentrations are elevated in women of advanced reproductive age undergoing ovulation induction. Fertil Steril 1997: 68: 607-612.

6. Seifer DB. Nafiolin F. Moving toward and earlier and better understanding of perimenopause. Fertil Steril 1996: 69: 387-388.

7. Seifer DB., Gardiner AC., Ferreira KA., Peluso JJ. Apoptosis as a function of ovarian reverse in women undergoing in vitro fertilization. Fertil Steril 1996: 66: 593-598.

8. Munne S., Cohen J. Chromosome abnormalities in human embryos. Hum Reprod Update 1998; 6: 842-855.

9. Hull MGR., Fleming CF., Hughes AO., McDermott A. The age related decline in female fecundity: a quantitative controlled study of implanting capacity and survival of individual embryos after IVF. Fertil Steril 1996: 65: 783-790.

10. Volaricik K.. Sheean L., Golfarb J et al. The meiotic competence of in vitro matured human oocytes is influenced by donor age: evidence that folliculogenesis is compromised in the reproductively age ovary. Hum Reprod 1998; 13: 150-160.

11. Brown JR., Liu HC., Sewitch KF., Rosewaks Z., Berkeley AS Variability of day 3 FSH levels in eumenorrheic women. J Reprod Med 1995; 40: 620-624

12. Roest J., Van Heudsen AM., Mous $\mathrm{H}$ et al. The ovarian response as a predictor for successful IVF treatment after the age of 40 years. Fertil Steril 1996; 66: 969-973.

13. Toner JP., Philput CB., Jones GS., Muasher SJ et al. Basal Follicule Stimulant Hormone levels is a better predictor of in vitro fertilization perfomance than age. Fertil Steril 1991: 55: 784-791.

14. Navot D., Rosenwaks Z., Margalioth EJ. Prognostic assesment of female fecundity. Lancet 1987; 2: 645-647.

15. Loumaye E.. Billion JM.. Mine JM et al. Prediction of individual response to $\mathrm{COH}$ by means of a clomiphene challenge test. Fertil Steril 1990; 53: 295-301.

16. Mukherjee T., Copperman AB., Lapinski R et al. An elevated day 3 FSH/LH ratio in the presence of a normal day 3 FSH predicts a poor response to controlled ovarian stimulation. Fertil Steril 1996: 65: 588593.

17. Padilla SL.. Smith RD., García J. The Lupron screening test. Tailoring the use of leuprolide acetate in ovarian stimulation. Fertil Steril 1991; 56: 79-83.

18. Fanchin R.. de Siegler D., Olivennes F, el al. EFORT test: a simple method and reliable screening test for detecting poor responders in IVF. Hum Reprod 1994; 9: 1607-1611.
19. Chang MY., Chiang CH., Chiu TH et al. The antral follicle count predicts the outcome of pregnancy in a $\mathrm{CHO} / \mathrm{IUI}$ programme. J Assist Reprod Genet 1998; 15: 12-17.

20. Collins W., Jurkovic D.. Bourne $T$ et al. Ovarian morphology, endocrine function and intrafollicular blood flow during the periovulatory period. Hum Reprod 1991; 6: 319-324.

21. Fanchin R., Harmas A., Benaoudia F et al. Microbial flora of the cérvix assessed at the time of embryo transfer adversely affects in vitro fertilization outcome. Fertil Steril 1998; 70: 866-870.

22. American Society for Reproductive Medicine. Revised American Society for Reproductive Medicine classification of endometriosis: 1996. Fertil Steril 1997; 67: 817-821.

23. Drake TS., O'Brien WF., Ramwell PW et al. Peritoneal fluid thromboxane 82 and 6-keto prostaglandyn $\mathrm{F} 1$ alpha in endometriosis. Am J Obstet Gynecol. 1981; 140: 401.

24. Marik J., Hulka J. Luteinized unruptured follicle syndrome: a subtle cause of infertility. Fertil Steril 1978; 29: 270.

25. Muscato JJ. Haney AF., Weinberg JB. Sperm phagocytosis by human peritoneal macrophages: a possible cause of infertility in endometriosis. Am J Obstet Gynecol 1982: 144: 503.

26. Avila LM.. Jara A.. Jiménez E.. Madero Jl. Correlation betwen the presence of anti-endometrial $\mathrm{IgG}$ antibodies and $\mathrm{Clq}$ deposit in endometrial tissue in patients with endometriosis. Hum Reprod 1997; 12: Abstr book 1: 193.

27. Cano F., Raigoza G., Posada N. Aspectos fisiopatológicos e inmunológicos de la endometriosis. Medicina Reproductiva 1997; 1: $8-14$.

28. Centers for Disease Control and Prevention. 1998 guidelines for treatment of sexually transmitted diseases. MMWR 1998; 47: 1-111.

29. Knutzen V.. Stratton C... Sheer G et al. Mock Embryo Transfer in early lutheal phase. Fertil Steril 1992; 57: 156-162.

30. Rosemberg E., Bronfenmaier S.. Olivieri MT. Consideraciones sobre la transferencia embrionaria. Medicina Reproductiva 1997; 1: 30-34.

31. Mansour R., Aboulgar M., Serour G. Dummy embryo transfer: a technique that minimizes the problem of embryo transfer and improves the pregnancy rate in human in vitro fertilization. Fertil Steril 1990; 54: $678-681$

32. Mayer J., Walker D., Jones E., Maloney M. et al. Significantly different pregnancy rate in a randomized study of two different transfer catheters and procedures. Abstracts of the Fifty second annual meeting of the American Society for Reproductive Medicine. November 2-6, 1996; S-211.

33. Nabi A., Awonuga A., Birch $\mathrm{H}$ et al. Multiple attempts at embryo transfer: does this affect in-vitro fertilization treatment outcome? Hum Reprod 1997: 12: 1188-1190.

34. Kruger TF., Acosta AA., Simmons KF et al. Predictive value of abnormal sperm morphology in in vitro fertilization. Fertil Steril 1988; 49: 112-117.

35. Kruger TF., Menkveld R., Stander Fet al. Sperm morphologic features as a prognostic factor in in vitro fertilization. Fertil Steril 1986; 46: $1118-1123$

36. Coetzee K., Kruger TF.. Lombard CJ. Predictive value of normal sperm morphology: a structured literature review. Hum Reprod Update 1998: 4: $73-82$. 
37. Aitken RJ., Irvine RS. Reliability of methods for assessing the fjertilizing capacity of human spermatozoa. En: Ombelet W, Bosmans E, Vandeput H, Vereecken A, Renier M, Hoomans E. (eds) Modern ART in the 2000s. New York: Parthenon Publishing Group, 1998; (8): 179-189.

38. Krausz C., Mills C., Rogers S et al. Stimulation of oxidant generation by human sperm suspensions using phorbol esters and formyl peptides: relationship with motility and fertilization in vitro. Fertil Steril 1994; 62: 599-605.

39. Bussen S., Zimmermann M., Schleyer M.. Steck T. Relationship of bacteriological characteristics to semen indices and its influence on fertilization and pregnancy rates after IVF. Acta Obstet Gynecol Scand 1997; 76: 964-968.

40. Jedrzejczak P., Szumala-Kalol A., Dydowics P et al. Usefulness of counting leukocytes and round cells in determination of bacterial infection of semen in infertile men. Gynekol Pol 1996; 11: 569-573.

41. Kruszewska D., Lembowics K., Tylewska-Wierzbanowska S. Possible sexual transmission of Q fever among humans. Clin Infec Dis 1996; 6: $1087-1088$.

42. Franken DR., Kruger TF, Menkveld R et al. Hemizona assay and teratozoospermia: increasing sperm insemination concentrations to enhance zona pellucida binding. Fertil Steril 1990; 54: 497-503

43. Oehninger S., Sayed E., Kolm P. Role of sperm morphology test in subfertility treatment strategies. En: Ombelet W, Bosmans E, Vandeput $\mathrm{H}$, Vereecken A, Renier M, Hoomans E. (eds) Modern ART in the 2000s. New York: Parthenon Publishing Group, 1998; (8): 25-36.

44. Oehninger S., Acosta AA., Morshedi Met al. Corrective measures and pregnancy outcome in in vitro fertilization in patients with severe sperm morphology abnormalities. Fertil Steril 1988; 50: 283-287.
45. Oehninger S., Sayed E., Kolm P. Role of sperm morphology test in subfertility treatment strategies. En: Ombelet W, Bosmans E, Vandeput H, Vereecken A, Renier M. Hoomans E. (eds) Modem ART in the 2000s. New York: Parthenon Publishing Group, 1998; (8): 25-36.

46. Oehninger S., Blackmore P., Morshedi $\mathrm{M}$ et al. Defective calcium influx and acrosome reaction (spontaneus and progresterona-induced) in spermatozoa of infertile men with severe teratozoospermia. Fertil Steril 1994; 61: 349-354.

47. Aitken RJ.. Irvine DS. Reliability of methods for assessing the fertilizing capacity of human spermatozoa. En: Ombelet W, Bosmans E, Vandeput H, Vereecken A, Renier M, Hoomans E (eds). Modern ART in the 2000s. New York. Parthenon Publishing Group, 1998; (8): 179-189.

48. Huszar G., Vigue L., Oehninger S. Creatine kinase immunocytochemistry of human sperm-hemizona complexes: selective binding of sperm with mature creatine kinase-pattern. Fertil Steril 1994; 61: 136-142.

49. Comhaire F., Zalata A., Mahmoud A et al. Doagnostic and therapeutic approach to moderate and severe male subfertility in 1995. Hum Reprod 1995; 10 suppl 1: 144-150.

50. Nagy ZP. Verheyen G., Liu J et al. Results of 55 intracytoplasmic sperm inyection cycles in the treatment of male-immunological infertility. Hum Reprod 1995; 10: 1775-1780.

51. Joesbury KA., Edirisinghe WR., Phiilips MR., Yovich JL. Evidence that male smoking affects the likelihood of a pregnancy following IVF treatment: application of modified cumulative embryo score. Hum Reprod 1998: 13: 1506-13. 